\title{
LBNO-DEMO (WA105): a large demonstrator of the liquid argon dual-phase TPC
}

Vyacheslav Galymov*†

Institut de Physique Nucléaire de Lyon, Villeurbanne, France

E-mail: vgalymoveipnl.in2p3.fr

The LBNO-DEMO (WA105 experiment) is a demonstrator of a dual-phase liquid argon timeprojection chamber concept. This prototype will have a $6 \times 6 \times 6 \mathrm{~m}^{3}$ (approximately $300 \mathrm{t}$ ) active volume and will allow to test industrial solutions developed for a construction of a large underground liquid argon detector for neutrino and antiparticle physics. The WA105 detector will be exposed to a well-defined beam of charged particles of different types and momenta to study and calibrate its performance. In these proceedings an overview of the WA105 detector design and its key features will be given. Implementation of the dual-phase detector in the context of the next-generation long baseline neutrino oscillation program will also be briefly discussed.

The European Physical Society Conference on High Energy Physics 22-29 July 2015

Vienna, Austria

* Speaker.

† On behalf of the WA105 collaboration 


\section{Introduction}

A liquid argon time projection chamber (LAr TPC) [1] is an attractive detector option for next generation of long-baseline neutrino oscillation experiments, nucleon decay searches, and Supernova neutrino detection. These application require a detector with active volume mass on a multi-kt scale and there has been extensive R\&D efforts throughout the world aimed at realizing this goal. In Europe, a fully engineered solution for a LAr TPC detector with a mass of $20 \mathrm{kt}$ to $50 \mathrm{kt}$ has been developed within the LAGUNA-LBNO design study. It is based on the GLACIER (Giant Liquid Argon Charge Imaging ExpeRiment) concept [2], a LAr TPC operated in dual-phase mode for charge readout.

The principal features of a LAr TPC with a dual-phase charge readout are illustrated in the left panel of Figure 1. A charged particle traveling through the liquid argon detector volume ionizes the medium. The ionization electrons are drifted up under the influence of an electric field to the gas-liquid boundary. They are then extracted from the liquid into the cold pure Ar vapour with a help of submerged grid of wires providing a local field of $2 \mathrm{kV} / \mathrm{cm}$. Once extracted, electrons in the gas pass through a high electric field in the holes of a $1 \mathrm{~mm}$ thick GEM or LEM (Large Electron Amplifier) where they are amplified following avalanche cascades. The resultant signal is collected on a $2 \mathrm{D}$ segmented anode. In addition to charge, ionizing particles also produce scintillation light in ultraviolet range $(128 \mathrm{~nm})$. This light can be detected with an array of cryogenic photo-multiplier tubes (PMT) and used to provide trigger signal and absolute time reference for the charge readout electronics.
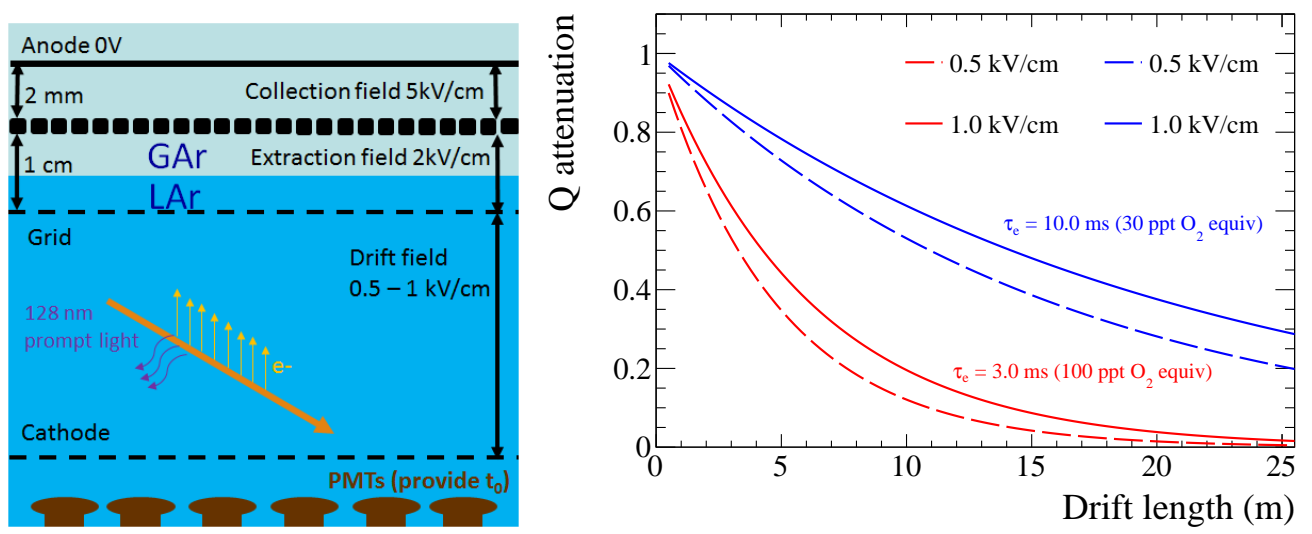

Figure 1: Left: Concept of a dual-phase LAr TPC (not to scale). Right: Attenuation of the electron survival probability as a function of drift distance for different electric field strengths and purity conditions; the latter is typically expressed as electron lifetime, i.e. the time it takes initial charge to decrease by a factor of $e^{-1}$.

Amplification of ionization charge in pure Ar gas, the key feature of the dual-phase LAr TPC concept, allows to achieve long drift distances by compensating electron losses in the liquid argon due to their attachment to the electronegative impurities (see the plot on the right in Figure 1). This, in turn, makes it feasible to build large and fully active detector volumes using well-established industrial technologies for storage and transport of liquid natural gas. In addition, the electron gain improves signal-to-noise ratio and lowers the energy threshold for particle detection enhancing the sensitivity to the low energy physics particularly measurements of the Supernova neutrino flux. 
The goal of the WA105 experiment [3] is to construct a large prototype dual-phase LAr TPC based on the LAGUNA-LBNO design in order to test all the principal components necessary for building a multi-kt scale detector. The detector will also be exposed to a well-defined charged particle test beam at CERN allowing to calibrate its performance, study energy resolution for hadronic and electromagnetic showers, and validate reconstruction algorithms. In the global context, the experiment will provide a critical feedback for the Deep Underground Neutrino Experiment (DUNE) - the planned next-generation long-baseline neutrino oscillation experiment.

\section{Overview of WA105 detector}

An overview of the WA105 detector is shown in Figure 2. The dimensions of the active volume are $6 \times 6 \times 6 \mathrm{~m}^{3}$, as outlined by the field cage structure, giving a total active mass of around $300 \mathrm{t}$. The beam pipe that delivers the charged particle beams is also visible in the figure.

Ionization electrons drift upwards toward the Charge Readout Plane (CRP). The nominal electric field strength is $0.5 \mathrm{kV} / \mathrm{cm}$ giving an expected electron drift velocity of around $1.6 \mathrm{~mm} / \mu \mathrm{s}$. Such field strength over $6 \mathrm{~m}$ drift corresponds to applying $300 \mathrm{kV}$ on the cathode. This is currently possible with commercially available power supplies (Heinzinger electronic $\mathrm{GmbH}$ ). In addition, an $R \& D$ program with the industry is envisioned to produce a power supply capable of delivering $600 \mathrm{kV}$ for the field strength of $1 \mathrm{kV} / \mathrm{cm}$.

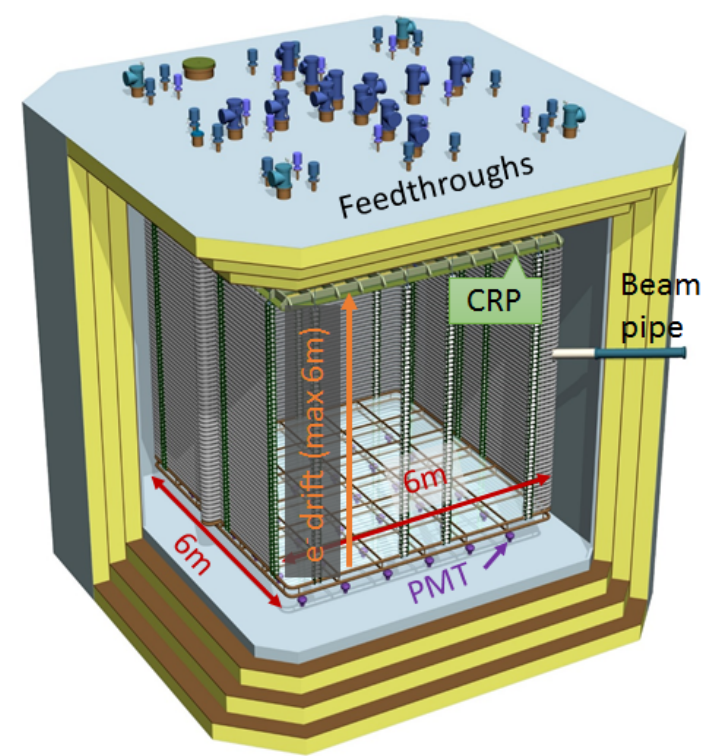

Figure 2: Overview of WA105 dual-phase LAr TPC detector.

After the drift, ionization charges are extracted, amplified, and collected at the CRP, which consists of four independent $3 \times 3 \mathrm{~m}^{2}$ sub-modules completely covering the active area of the detector $\left(6 \times 6 \mathrm{~m}^{2}\right)$. Each of these units is built from $50 \times 50 \mathrm{~cm}^{2}$ fully active LEMs and anodes, shown in Figure 3, that operate in ultra-pure cold Ar vapour above the liquid surface.

The LEM is a $1 \mathrm{~mm}$ thick copper-clad epoxy PCB (printed circuit board) with $500 \mu \mathrm{m}$ holes drilled in a honeycomb pattern with a pitch of $800 \mu \mathrm{m}$. The holes act to confine UV photons 


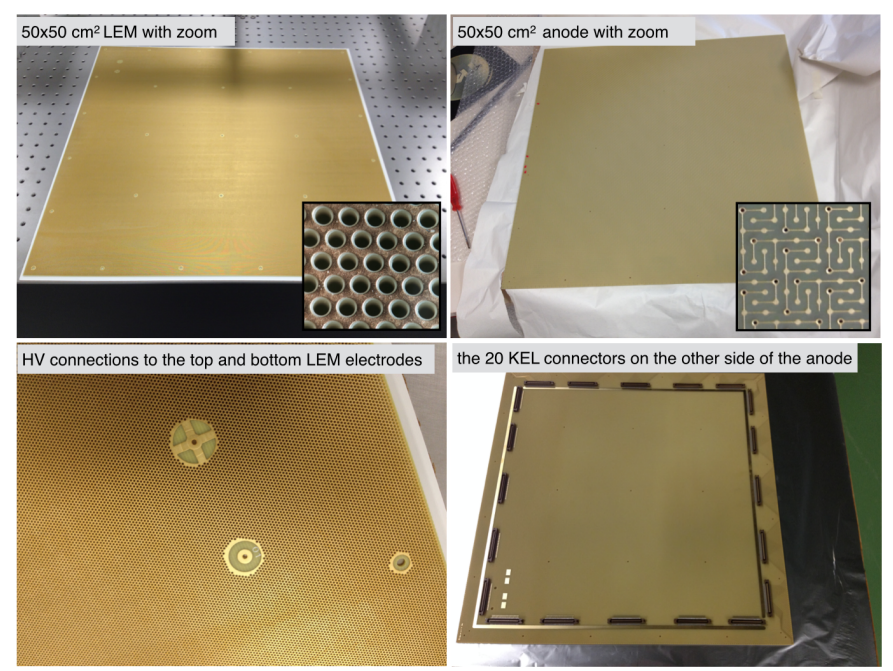

Figure 3: Top: pictures of the LEM and anode with a microscopic views. Bottom: close up of the HV connectors on the LEM and back view of the anode.

produced during avalanche and thus suppressing photon feedback. The shape of the holes, their size as well as the overall PCB thickness have been optimized to maximize the gain and improve operation stability [4]. Maximum gain of $\sim 200$ has been reached, while the stable gain of at least 15 has been shown to be feasible.

The anode is multi-layer PCB with a strip pitch of $3.125 \mathrm{~mm}$ in both $x$ and $y$ views. The strip layout has been optimized experimentally to ensure the charge collection is the same between the two views and is independent of the azimuthal angle of charged particles [5]. The anode strips within each of the sub-unit are bridged at the back (see Figure 3) to form continuous strips of $3 \mathrm{~m}$ long thus giving a total of 1920 (or 960 per view) readout channel per the $3 \times 3$ module.

Each $3 \times 3$ module acts as an independent detector with its own suspension system, slow control, and channel readout. The extraction grid is mounted on the frame $10 \mathrm{~mm}$ below the LEM lower surface. It is built from $100 \mu \mathrm{m}$ stainless steel wires tensioned in both $x$ and $y$ with the pitch identical to that of the anode to avoid charge shadowing effects. The suspension system adjusts each module such that the extraction grid sits $5 \mathrm{~mm}$ below the liquid level. The required precision for the CRP position with respect to the liquid level is $1 \mathrm{~mm}$.

The signal lines are extracted from top of the anode deck via a system of feedthrough chimneys. To minimize the cable length and resulting noise, analog pre-amplifiers are placed at the bottom of the chimneys as close as possible to the CRP. The key aspect of this system is that the chimneys are physically isolated from the cryostat inner volume allowing access to the front-end electronics for potential maintenance without opening of the detector.

The front-and analog pre-amplifiers are based on CMOS technology and have a very low power consumption (18 $\mathrm{mW} /$ readout $\mathrm{ch}$ ). Due to low ambient temperature of $110 \mathrm{~K}$-maintained constant inside each chimney using a heat exchanger with liquid nitrogen-the noise in these devices is minimized. The digital electronics is housed in $\mu$ TCA crates, which are mounted on the top of the signal feedthrough chimneys. The charge signals are digitized at $2.5 \mathrm{MHz}$ sampling rate for total time of $4 \mathrm{~ms}$, which fully covers the time needed for charge to drift $6 \mathrm{~m}$ in $0.5 \mathrm{kV} / \mathrm{cm}$ field. 
The scintillation light readout is accomplished with an array of 8 inch cryogenic PMTs (Hamamatsu RD5912mod2) placed below the cathode (Figure 2) with one detector per $1 \times 1 \mathrm{~m}^{2}$. The ultraviolet scintillation light is shifted into visible spectrum to match the response of the photocathode using tetraphenyl butadiene (TPB), an organic wavelength shifter. The TPB coating could either be applied directly on the PMT glass window or a transparent acrylic plate mounted above the photo-cathode surface. Both options are being studied at the moment.

While for deep underground operation the light signal would provide the absolute event time and possible trigger signal, in the case of the WA105 detector, operated on the surface, the light readout system has to also ensure that cosmic ray background muons could be efficiently tagged within a given beam event. For these reasons two modes of operation are envisioned for the light readout electronics. In the first mode, the PMTs are continuously read within \pm 4 ms time window with respect to the beam trigger with a coarse time sampling (possibly $2.5 \mathrm{MHz}$ similar to charge readout) to identify cosmic ray activity in the detector. In second mode, outside of the beam spill window, a self-triggering system based on a custom on-board (PARISROC2) ASIC is used to acquire short time samples, on the order of ten $\mu \mathrm{s}$, with a fine sampling rate of $40 \mathrm{MHz}$ in order to analyze LAr scintillation and PMT performance in greater detail.

\section{Operation plan and schedule}

The WA105 detector will be housed in the extension of the EHN1 test beam facility currently under construction at the CERN Prévessin site. It will be exposed to secondary beams of different types and momenta. In particular electrons, muons, and pions of both positive and negative charge, and momenta in the range of $0.5-10 \mathrm{GeV} / \mathrm{c}$ will be studied. The expected beam event rate is 100 $\mathrm{Hz}$. The planned start of data taking campaign is the end of 2017.

\section{Dual-phase TPC for DUNE}

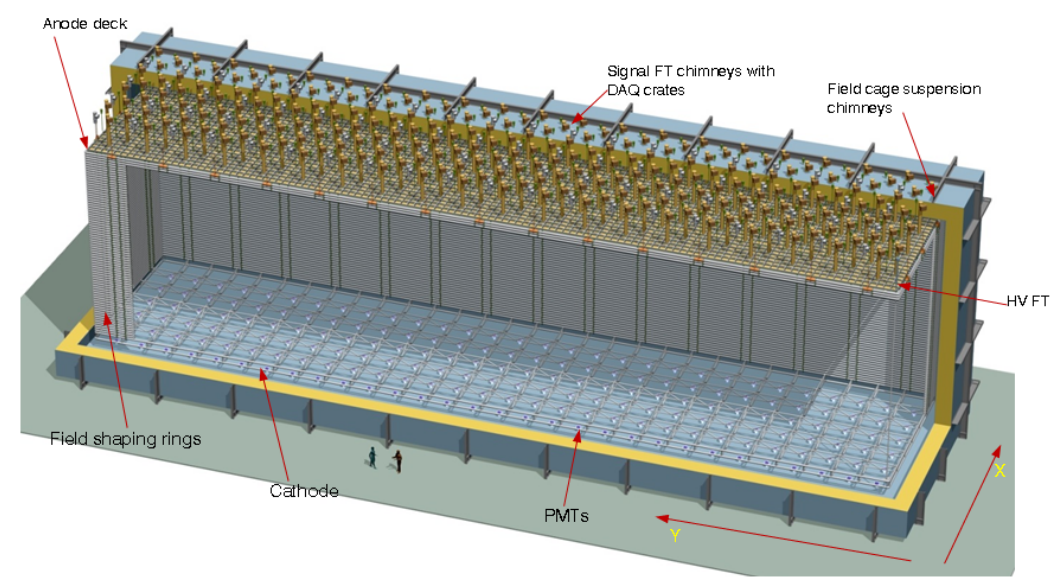

Figure 4: The DUNE dual-phase LAr TPC detector (with the field cage partially open for clarity) with cathode, PMTs, field cage, and anode plane with service chimneys.

The success of the WA105 experiment would pave the way for the implementation of a dualphase LAr TPC in the DUNE experiment. DUNE has a multifaceted physics program covering long 
baseline neutrino oscillations - with an particular aim of discovering the neutrino mass hierarchy and leptonic CP violation-, nucleon decay searches, and detection of low-energy neutrinos from Supernova explosions. An underground detector complex with a total active LAr mass of $40 \mathrm{kt}$ is planned to be constructed in the Sanford underground research facility in South Dakota, USA. Four cryostat each housing $10 \mathrm{kt}$ of active mass are envisioned. In Figure 4, a design concept of a cryostat with a $10 \mathrm{kt}$ dual-phase LAr TPC is shown. The overall dimensions of the active volume are $12 \times 60 \times 12 \mathrm{~m}^{3}$ for corresponding to the width, length, and height (distance from the cathode to anode) respectively. The strength of the drift electric field is $0.5 \mathrm{kV} / \mathrm{cm}$. To achieve this field $600 \mathrm{kV}$ has to applied to the cathode. The solution for such high voltage power supply and the necessary feedthrough system will be developed and tested as part of the WA105 program.

Although covering a much larger are than in the case of WA105, DUNE CRP is composed of individual $3 \times 3$ units as in the prototype detector. The scintillation light is collected with an array of 180 PMTs positioned at the bottom of the cryostat (see Figure 4 ) each within $4 \times 4 \mathrm{~m}^{2}$ area. The PMT are also planned to be identical to the ones used in WA105.

\section{Conclusions}

The WA105 experiment plans to construct and operate a dual-phase LAr TPC with a $300 \mathrm{t}$ of active volume. The detector will be built in the test beam facility at CERN where it will be exposed to well-defined particle beams allowing to study its performance, understand calorimetric response to electromagnetic and hadronic showers, and develop and fine-tune reconstruction algorithms. The success of the WA105 program will give an opportunity to build a multi-kt scale dual-phase LAr TPC within the future long-baseline neutrino oscillation program.

\section{Acknowledgments}

The author wishes to thank the LabEx Lyon Institute of Origins (ANR-10-LABX-0066) of the Université de Lyon for its financial support within the program "Investissements d'Avenir" (ANR11-IDEX-0007) of the French government operated by the National Research Agency (ANR).

\section{References}

[1] C. Rubbia, The Liquid Argon Time Projection Chamber: A New Concept for Neutrino Detectors, CERN-EP-INT-77-08 (1977)

[2] A. Rubbia, Experiments for CP violation: A Giant liquid argon scintillation, Cerenkov and charge imaging experiment? (2014) [hep-ph/0402110]

[3] L. Agostino et al., LBNO-DEMO: Large-scale neutrino detector demonstrators for phased performance assessment in view of a long-baseline oscillation experiment, CERN-SPSC-2014-013, SPSC-TDR-004 (2014) [arXiv: 1409.4405 ]

[4] C. Cantini et al., Performance study of the effective gain of the double phase liquid Argon LEM Time Projection Chamber, JINST 10 (2015) 03017 [arXiv: 1412.4402]

[5] C. Cantini et al., Long-term operation of a double phase LAr LEM Time Projection Chamber with a simplified anode and extraction-grid design, JINST 9 (2014) 03017 [arXiv: 1312 . 6487] 